\title{
Analysis of Paraffin Wax as a Phase Change Material
}

\author{
P Vinod Kumar Naidu** and Ajay M Nair ${ }^{\#}$ \\ ${ }^{\wedge}$ Department of Mechanical Engineering, Chaitanya Bharathi Institute of Technology, Hyderabad, India \\ \#Department of Mechanical Engineering, Toc H Institute of Science and Technology, Ernakulam, India
}

Received 01 Nov 2017, Accepted 01 Jan 2018, Available online 09 Jan 2018, Vol.8, No.1 (Jan/Feb 2018)

\begin{abstract}
As the energy demand increases and with the conventional fossil fuels depleting, the energy crisis in the near future is bound to worsen. Moreover fossil fuels have adverse effects on the environment so it is required to switch on to renewable sources of energy to meet the energy requirements. Especially solar energy is abundantly available but it is intermittent, unpredictable, and available only during the day. An efficient thermal energy storage system is required for storing the surplus energy available during light hours so that it can be used during nights. This paper is focused on the charging and discharge analysis of Paraffin wax (melting temperature of $58-60^{\circ} \mathrm{C}$ ) which is used as phase change material in thermal energy storage system. To analyse the performance of Paraffin wax, a simple and economical experimental setup has been constructed that consists of solar parabolic concentrator, hot water bath, water pump, test section, insulation boxes, T type thermocouple and Data acquisition system. In the experiment conducted, the water which gets heated due to the solar parabolic concentrator charges the Paraffin wax in the test section. Once the Paraffin wax gets fully charged, it is taken out and kept in the insulation box .The temperature variation with time while charging and discharging is recorded. It is found that Paraffin wax took 3 hours and 7 minutes for getting completely charged and it took 15 hours 28 minutes for discharging i.e. there was a temperature drop of $20.86^{\circ} \mathrm{C}$ from the initial temperature of $69.9^{\circ} \mathrm{C}$
\end{abstract}

\section{Keywords: Change Materials (PCMs), Chagrining, Discharging, Storage period}

\section{Introduction}

Energy is the backbone of all human activities on the earth and globalization and rapid economic growth has led to a rapid increase energy demand. Fossil fuels have been main source of energy for human need but it reserves are running out and fossil fuel causes environmental such as global warming and melting of ice in south and north poles. Therefore renewable energies like solar energy, bio energy wind energy, geothermal energy, tidal energy are investigated, even though solar energy is abundant available throughout the year in the most part of the earth, they are intermittent in nature as they are not available all the time, this intermittent problem can be solved by suitable energy storage. PCM based thermal energy storage is effective in nature.

Jian-you et al studied effect of inlet temperature and flow rate of heat transfer fluid HTF (including the hot HTF and cold HTF) on the storage unit performance experimentally for the numerical model validation, $N$-Hexacosane $\left(56.3{ }^{\circ} \mathrm{C}, \mathrm{LH}-61 \mathrm{kcal} / \mathrm{kg}\right)$ as a PCM. The thermal energy storage unit composes of a triplex concentric tube filled with PCM in the middle

*Corresponding author's ORCID ID: 0000-0002-5835-4608 DOI: https://doi.org/10.14741/ijcet.v8i01.10888 channel, with hot HTF flowing through the outer channel during the charging process and cold HTF flows through the inner channel during the discharging process. Numerical and experimental results showed that every $2{ }^{\circ} \mathrm{C}$ enhancement of inlet temperature of cold HTF results in an average heat recovery rate increase of $0.042 \mathrm{~kW}$. Hoshi et al classified the phase change materials based on their melting points temperature, such as low temperature phase change materials are the material having Melting Point temperature less than $220^{\circ} \mathrm{C}$, medium temperature phase change materials are the material having Melting Point temperature up to $420^{\circ} \mathrm{C}$ and high temperature phase change materials are the material having Melting Point temperature greater than $420^{\circ} \mathrm{C}$.

Castell et al presented an experimental study of a PCM storage unit for cold storage applications. The study demonstrated that coil in storage unit designs are effective to delivering a constant outlet temperature and effective heat transfer with large surface areas. Halawa et al have studied numerically the thermal performance of an air based PCM storage unit which uses Chloride Hexahydrate ( $\mathrm{CaCl} 2,6 \mathrm{H} 2 \mathrm{O})$ $\mathrm{MP}$ at $28{ }^{\circ} \mathrm{C}$ for space heating application and the effects of temperature during the melting /freezing process and found out the high mass flow rate, 
increases heat transfer thus reduces the melting/freezing duration on other hand as slabs thickness increase, heat transfer rate decreases and the change in PCM dimensions when the PCM surface area and mass is kept constant has no effects on the outlet temperature and rate of the heat transfer. Lopez et al. et al was studied numerically and experimentally the thermal energy storage using PCM in the air heat exchanger. The model can be used for both heating and cooling in buildings applications. Li Yongai and Li Shuli et al experimentally investigated of the solar chimney with application of PCM. With three different heat flux i.e. $700 \mathrm{~W} / \mathrm{m}^{2}, 600 \mathrm{~W} / \mathrm{m}^{2}$ and $500 \mathrm{~W} / \mathrm{m}^{2}$.The absorber surface temperature variations for the three heat fluxes are the same during the phase change transition period. PCM based solar chimney shows a satisfied and should be studied furthermore with lower heat fluxes, due to the complicated heat transfer characteristics of the PCM. Al-Kayie et al experimentally investigated the performance of a solar water heater at different inclination angles i.e. $10.20,30$ degrees with three different cases, one without thermal energy storage secondly with thermal energy storage of Paraffin wax , and the third case is of thermal energy storage with copper nano composites .water circulation rate is kept constant of $0.5 \mathrm{~kg} / \mathrm{min}$, results indicated that the enhancement of performance of the system by using thermal energy storage but there is not much enhancement recorded by using copper nano composites but the author recommended for investigation of the experiment with different flow rates. Mahfuz et al. Experimental study performance for solar water heating incorpated with Paraffin wax based shell and tube thermal energy storage system and determined the total life cycle cost, energy and exergy efficiencies of the thermal energy storage system at different flow rate of heat transfer fluid and found that with increase of flow rate the total life cycle cost decreases. Kabeel A E and Abdelgaied Mohamed et al studied experimentally the effect of PCM on the behaviour of the solar still with PCM. They compare the productivity of conventional solar still and solar still with PCM. The experimental results showed that the solar still with PCM was superior compared to the conventional solar still, the daily freshwater productivity (67\%-68.8\% improvement)

Many works had been done with Paraffin wax as PCM's but very little focus were there on the PCM's based solar thermal energy storage so in the present work charging of the Paraffin wax PCM is done by solar energy and charging and discharging period analysis of Paraffin wax is done in a simple experimental setup

\section{Experimental Set up}

The aim of the experimental work is to investigate the performance of PCM based thermal energy storage with the solar concentrator dish. In the present the Paraffin is used as which is available in different grades, here the melting temperature is $58-60^{\circ} \mathrm{C}$, the
Paraffin wax is shown in figure 1, The .schematic diagram of the experimental setup is shown in figure 2 .
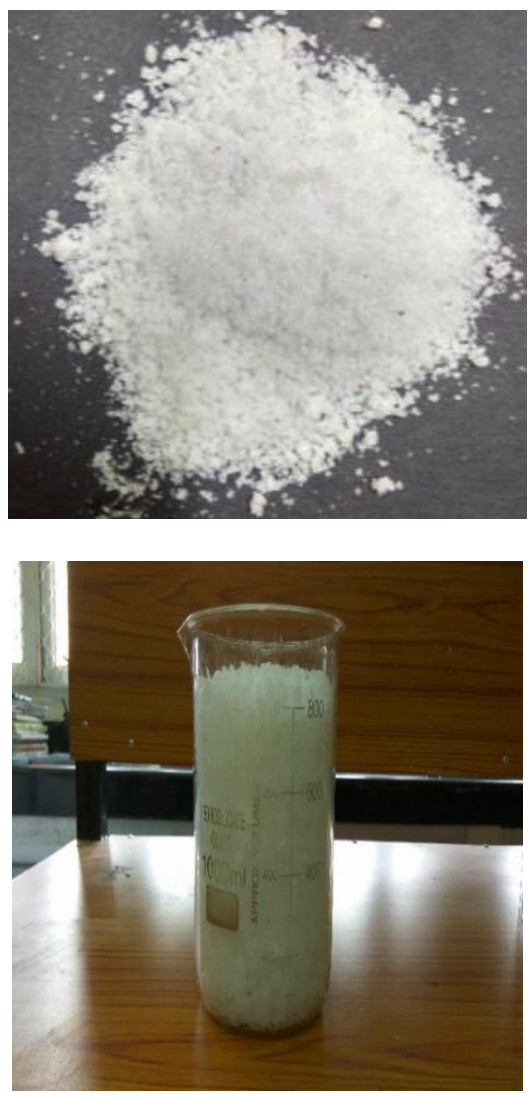

Fig 1 Pure Paraffin wax

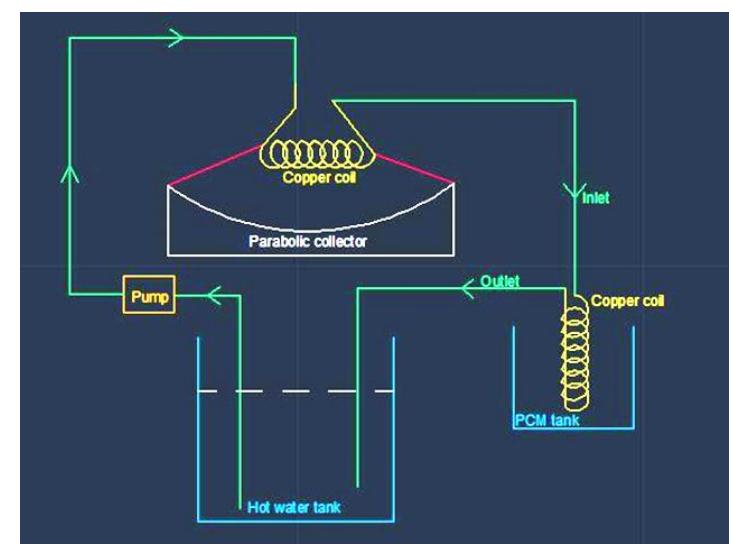

Fig 2.Schematic diagram of the experimental setup

The experimental set up consists of hot insulated water bath which is having multi-layer insulation like initially the water bath is painted with black colour on the both sides of it, on that cardboard (black paint is applied on it) over that thermocol covered with Aluminium foil is installed and at as last layer of insulation again cardboard with black painted is installed. Parabolic solar concentrator dish, which is made up of Aluminium sheet of focal length of $50 \mathrm{~cm}$ and a multi coiled $3 / 8$ in copper coil is placed at the focus so that the most of the thermal radiation is concentrated at the copper coil. Water pump (model star RS25/6 WILO 
Company Germany, capacity $800-3400$ lph, head of 2-5 m) PCM (Paraffin wax) cylinder, copper helical coil heat exchanger made up of $1 / 4$ in diameter copper tube, the helical coil having $8 \mathrm{~cm}$ outer diameter and length of $10 \mathrm{~cm}$, T Type thermocouple, Data acquisition system (Agilent 34972A LXI Data Acquisition/Switch Unit), Insulation boxes for storage which is having similarly insulation like hot water bath. Photograph of the experimental set up is shown in figure 3 .

Hot water from the parabolic solar concentrator dish is flow to the testing section where the Paraffin wax PCM is kept and the hot water flows through the copper helical coil heat exchanger charges the Paraffin wax and the water reaches to hot water bath where the water is collected and then it is pumped back to solar concentrator dish through pump and once the Paraffin PCM is get fully charged, it is taken out from the testing section and kept in the insulation boxes for the discharge and $\mathrm{T}$ type thermocouple is kept two at charging container and storage insulation container to record the variation of temperature of Paraffin wax at various interval of charging and discharging
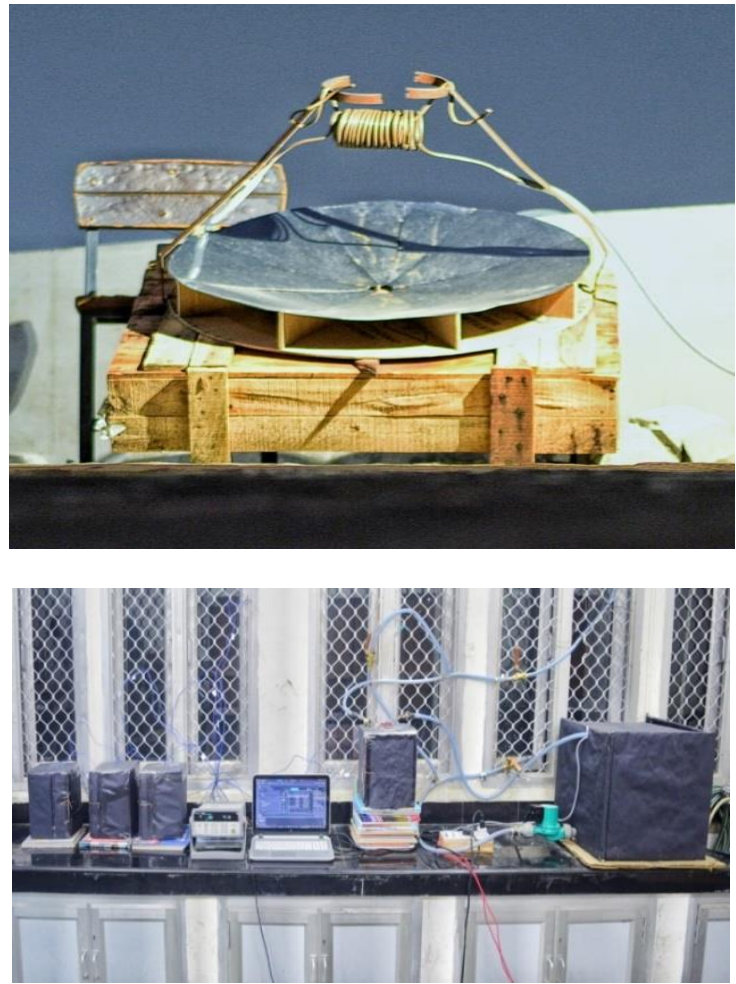

Fig 3 Photograph of the experimental set up

Table 1 Properties of Paraffin wax

\begin{tabular}{|c|c|c|c|c|}
\hline 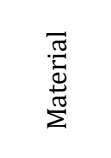 & 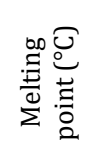 & 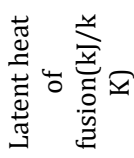 & 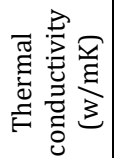 & 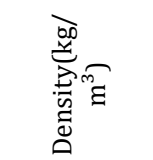 \\
\hline $\begin{array}{c}\text { Paraffin } \\
\text { Wax }\end{array}$ & $58-60$ & 189 & $\begin{array}{c}0.21 \\
(\mathrm{~s})\end{array}$ & $\begin{array}{l}795\left(1,70^{\circ} \mathrm{C}\right) \\
920\left(\mathrm{~s}, 20^{\circ} \mathrm{C}\right)\end{array}$ \\
\hline
\end{tabular}

\section{Results and Discussions}

Paraffin wax during charging and discharging

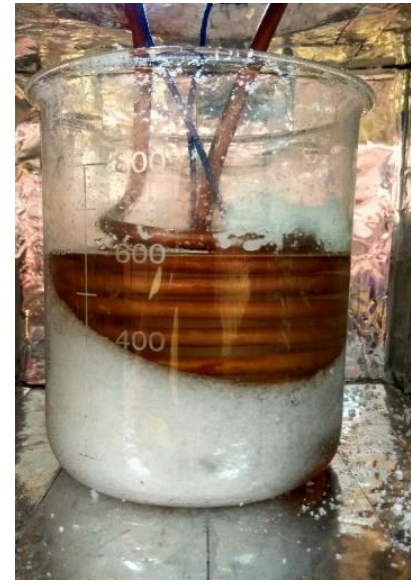

(a)

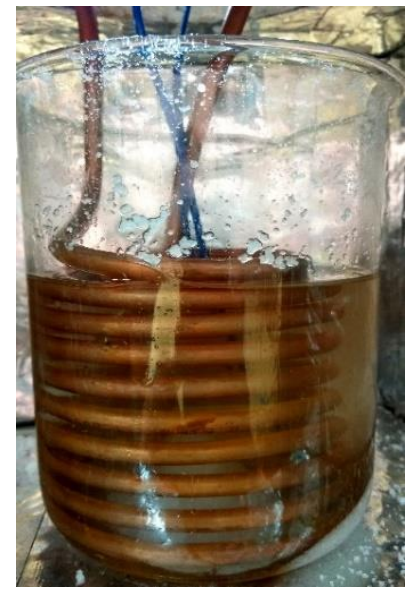

(b)

Fig 4(a)-(b) Condition of Paraffin wax at different stages of charging

During experimentation it is found that pure Paraffin wax took $3 \mathrm{~h} 5 \mathrm{~min}$ for complete charging and the end of complete charging the Paraffin wax attained a temperature of $70.2965{ }^{\circ} \mathrm{C}$ the variation of PCM temperatures with time during charging of pure Paraffin wax is shown in the graph.

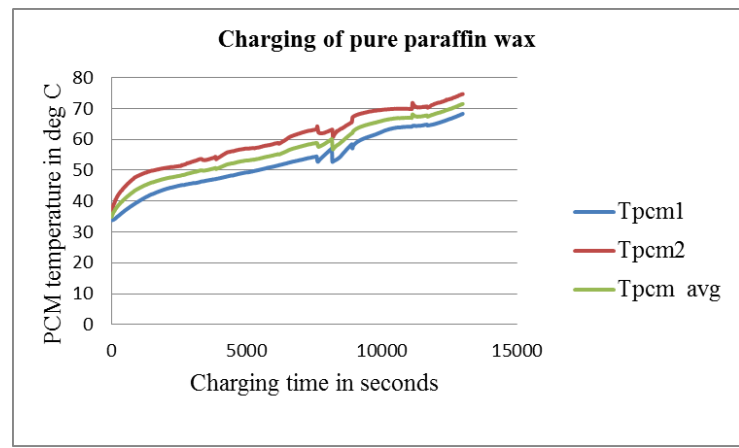

(a)

Fig 5(a) PCM temperature vs charging time pure Paraffin wax 


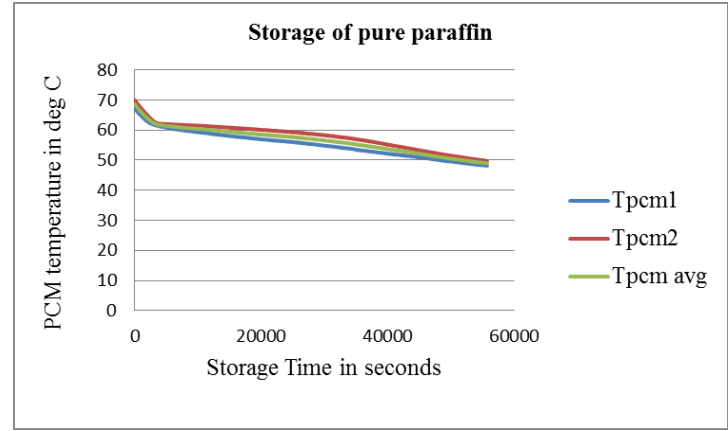

(b)

Fig5 (b) PCM temperature vs storing time pure Paraffin wax

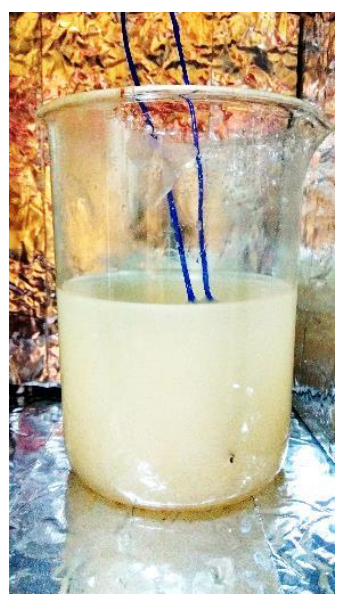

Fig 6 Condition of Paraffin wax during discharging

For storing period analysis Paraffin wax sample were kept in the storage box for 15 hours and 28 minutes and the variation in temperature was analysed. The temperature drop of pure Paraffin wax during at the end of 15hours and 28 minutes of storage was found to be $49.0355^{\circ} \mathrm{C}$ the variation of PCM temperatures with time during storing of pure is shown above

\section{Conclusions}

The performance analysis of pure Paraffin was done and this work presents the experimental study of the charging and discharging process of the LHTES. The following conclusions can be drawn from the results:
During the charging process, the Paraffin wax took around 3 hours and 5minutes and the discharge process comparatively long duration than that of charging process i.e. 15 hours and 28 minutes and still the temperature is around $49.0355^{\circ} \mathrm{C}$ and still the a lot of energy is stored in the sample as the storage duration prolongs the sample releases the heat energy In order to improve the performance of the Paraffin wax by adding suitable amount of micro or nano particles in to it, the care should be taken that the micro and nano particles disperses homogenously throughout the sample otherwise the micro or nano particle settle at the bottom during the experimentation

\section{References}

Jian-you Long (2008), Numerical and Experimental Investigation for Heat Transfer in Triplex Concentric Tube with Phase Change Material for Thermal Energy Storage, Solar Energy,Vol. 82, pp. 977-985.

Hoshi A, Mills DR, Bittar A, Saitoh TS (2005) Screening of high melting point phase change materials (PCM) in solar thermal concentrating technology based on CLFR. Sol Energy;79:332-9.

Castell, M. Belusko, F. Bruno, L.F. Cabeza (2011), Maximisation of heat transfer in a coil in storage unit PCM cold storage system, Appl. Energy 88, 4120-4127.

Halawa E. and Saman W. (2011), Thermal Performance Analysis of A Phase Change Thermal Storage Unit for Space Heating", Renewable Energy, Vol. 36, pp. 259-264.

J.P.A. Lopez, F. Kuznik, D. Baillisc, J. Virgone (2013), Numerical modeling and experimental validation of a PCM to air heat exchanger, Energy Build. 64, 415-422.

Li Yongcai, Li Shuli, Experimental study on thermal performance of a solar chimney combined with PCM, Applied Energy 114 (2014) 172-178.

Al-Kayiem H Hussain, Saw C. Lin (2014), Performance evaluation of a solar water heater integrated with a PCM nanocomposite TES at various inclinations, Solar Energy 109, 82-92

Mahfuz M H, Anisur M R, Kibria M A, Saidur R (2014), Metselaar I H S C, Performance investigation of thermal energy storage system with Phase Change Material (PCM) for solar water heating application, International Communications in Heat and Mass Transfer 57, 132-139

Kabeel A E, Abdelgaied Mohamed (2016), Improving the performance of solar still by using PCM as a thermal storage medium under Egyptian condition, Desalination 383, 22-28. 